\title{
Abstracts From the NCCN 22nd Annual Conference: Improving the Quality, Effectiveness, and Efficiency of Cancer Care ${ }^{\mathrm{TM}}$
}

The following abstracts were accepted for presentation at the NCCN 22nd Annual Conference: Improving the Quality, Effectiveness, and Efficiency of Cancer Care ${ }^{\mathrm{TM}}$ General Poster Session at the Rosen Shingle Creek resort in Orlando, Florida, on March 23 and 24, 2017. Additional abstracts will be available at JNCCN.org.

\section{Best Practices in Implementation and Use of Clinical Practice Guidelines \\ AB2017-1. Investigation of Gelclair Bioadherent Oral Gel: Novel Analyses and a Proposed Study on Oral Mucositis Development and Management in Comparison to Magic Mouthwash/Standard of Care \\ Mark S. Chambers, DMD, MSa; Katharine Ciarrocca, DMD, MSEd ${ }^{\mathrm{b}}$; Mary Kay Delmedico, $\mathrm{PhD}^{c}$; and Andrea True Kelly, $\mathrm{PhD}^{c}$

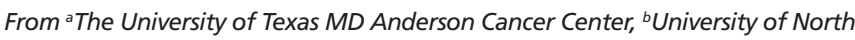 Carolina School of Dentistry, and 'Midatech Pharma US Inc.}

Background: Oral mucositis (OM) is a debilitating side effect of chemotherapy and radiotherapy (RT) in patients receiving myeloablative conditioning before stem cell transplantation (SCT). OM may result in opiate use, need for total parenteral nutrition (TPN), secondary infections, decreased quality of life, and reduction/interruption of therapy, ultimately compromising outcomes and increasing costs. Magic mouthwash (MMW) is frequently used for OM despite recommendations against its use due to limited efficacy and adverse effects, including dysgeusia, numbness, burning, or aspiration. Gelclair (GEL) is a well-tolerated, alcohol-free, bioadherent, oral/topical gel containing polyvinylpyrrolidone, sodium-hyaluronate, and glycyrrhetinic acid (from licorice root) broadly indicated for the management of oral lesions, including OM. Promising results on pain control and reductions in analgesics, incidence/severity of $\mathrm{OM}$, and infection have been reported in patients receiving chemotherapy and RT. Additional randomized controlled trials with a relevant active comparator could support these positive findings. Methods: The purpose of this blinded, randomized, controlled trial in SCT recipients receiving myeloablative conditioning is to determine the impact of GEL versus MMW/standard of care (SOC), when initiated during conditioning or after $\mathrm{OM}$ is noted, following a standardized oral care protocol. Subjects will be randomized (1:1:1) to initiate GEL for up to 28 days on conditioning day 1 or after diagnosis of grade $1 \mathrm{OM}$ versus MMW or SOC. A blinded clinician will conduct daily assessments and determine the incidence, time to onset, and duration of any or severe OM; overall mouth/throat pain; duration of effect; healthcare resources utilized; and subject burden associated with $\mathrm{OM}$, such as number of treatments per day, opiate use, TPN, infection, and impact on activities of daily living. Results: New hypothesis-generating analyses of existing data sets show that in patients with chemotherapy- and RT-induced OM, by 3 and 5 hours posttreatment, only $10 \%$ and $35 \%$ of GEL patients required a second dose versus $27 \%$ and $58 \%$ of MMW patients, respectively $(P=.037$ or $P=.034$, respectively), suggesting that GEL controls OM-related pain longer than MMW. Conclusions: GEL controlled chemotherapy- and RT-induced OM symptoms longer than MMW, and the randomized/blinded study under development at leading US cancer centers will address the impact of GEL on the incidence and severity of $\mathrm{OM}$ and its related symptoms, complications, and costs in SCT recipients receiving myeloablative conditioning relative to SOC.

\section{AB2017-2. Optimizing Posttreatment Surveillance in Patients With Locally Advanced Non-Small Cell Lung Cancer After Definitive Chemoradiotherapy \\ George Daniel Grass, MD, PhD; Arash O. Naghavi, MD, MS; \\ Yazan A. Abuodeh, MD; Bradford A. Perez, MD; \\ and Thomas J. Dilling, MD, MS \\ From H. Lee Moffitt Cancer Center and Research Institute}

Background: Although advancements in treatment have improved outcomes in non-small cell lung cancer (NSCLC), little evidence is available to guide oncologists in the manner of surveillance after definitive chemoradiation (CRT) in locally advanced NSCLC. Currently, the NCCN Guidelines recommend at least biannual surveillance imaging in the first 2 years and then annual followup afterward. This study evaluates time-based events of relapse after CRT to identify optimal posttreatment surveillance intervals. Methods: The records of 211 patients with stage III NSCLC treated with definitive CRT between 2005 and 2014 were retrospectively analyzed. Patient, tumor, and treatment characteristics were abstracted from the chart. Local, regional, and distant failure rates were assessed from the end date of radiotherapy (RT). Cumulative event tables were used to categorize failure events 
NCCN 22nd Annual Conference Abstracts

into posttreatment follow-up intervals. Patients with disease relapse were further stratified by presenting with or without recurrence-related symptoms before identification on follow-up imaging. Event rates were estimated with the Kaplan-Meier method and compared with log-rank tests. Predictors of outcome were evaluated with chi-square analysis. Results: Median follow-up was 18 months (range, 3-126 months), and the median patient age was 63 years. Equal proportions of patients had IIIA or IIIB disease, and 22\% were current smokers; $94 \%$ received platinum-doublet chemotherapy, and all received concurrent RT. A total of $64 \%$ had disease relapse and, of these, $74 \%$ experienced recurrence within 12 months of finishing RT. Among those who did not experience recurrence at $\leq 12$ months, $16 \%$, $6 \%$, and $<5 \%$ experienced recurrence during 12 to 24 , 24 to 36 , and $>36$ months of follow-up, respectively. The median onset of relapse was more rapid in patients with stage IIIB versus IIIA disease (8.1 vs 14.9 months; $P=.02) ; 56 \%$ had relapse-related symptoms before identification on follow-up imaging. No difference was seen in overall onset of relapse between those with or without symptoms, although those with symptomatic local failure had an earlier median onset ( 7.2 vs 16.4 months; $P=.04)$. Current smokers more frequently had symptomatic local failure than nonsmokers $(P=.003)$. Conclusions: We found that in patients with stage III NSCLC who experienced disease relapse after CRT, $75 \%$ experienced recurrence within 1 year, whereas the risk of recurrence after the first year was low. Prior symptoms seemed to have little relationship to the onset of relapse. Our study supports more aggressive surveillance imaging in the first year after CRT, with subsequent protraction thereafter.

\section{AB2017-03. Pilot Results of Consolidated Screening Tool for Distress and Supportive Oncology Needs \\ Christine B. Weldon, MBA; ; Nancy Vance, BA; \\ Amy Scheu, MSH' ; Lauren Allison Wiebe, MD'; Shelly S. Lo, MD'; \\ Catherine Deamant, MD; Betty Roggenkamp, BA; \\ Urjeet Patel, MD; ; Paramjeet Khosla, MD \\ Patricia A. Robinson, MDd; Ana Gordon, AM, LCSW; \\ Frank J. Penedo, PhDi; James Gerhart, $\mathrm{PhD}^{\mathrm{c}}$; Teresa Lillis, $\mathrm{PhD}$; \\ William Dale, MD PhD; \\ Rosa Berardi, BA'; and Julia R. Trosman, PhD, MBA' \\ From ${ }^{a}$ Northwestern University Feinberg School of Medicine, ${ }^{b}$ Advocate at Home, 'Rush University, 'Loyola University Medical Center, eJourney Care, fOn the Side Design, ${ }^{g}$ Cook County Health and Hospitals System, h' Cancer Care Center, 'University of Illinois Hospital \& Health Sciences System, iRobert H. Lurie Comprehensive Cancer Center of Northwestern University, "University of Chicago, and 'The Coleman Foundation}

Background: The Institute of Medicine (IOM) 2013 report recommends that supportive oncology care start at cancer diagnosis; the Commission on Cancer (CoC) Standard 3.2 requires distress screening and indicated action. There are many screening tools available, no- tably the NCCN Distress Thermometer and Problem List. However, screening tools are not standardized across institutions and often address only a portion of patients' supportive oncology needs. Methods: A collaborative of $\geq 100$ clinicians, funded by The Coleman Foundation, developed a patient-centric consolidated screening tool based on validated instruments, including 39 concerns adapted from the NCCN Distress Thermometer and Problem List, IOM report, and $\mathrm{CoC}$ standards. The tool also includes validated measures: the Patient Health Questionnaire-4 (PHQ-4) for anxiety and depression, and the Patient-Reported Outcomes Measurement Information System short forms for pain, fatigue, and physical function. The screening tool was piloted at 2 large community cancer centers. Results: Among 170 patients (69 men, 75 women, 26 not specified), an average of 4.8 supportive care concerns were reported. The most common responses were, "I want to better understand my prognosis or long-term outcome" (33\%); not finding "strength in my faith and beliefs" (33\%); "I want to better understand my cancer diagnosis or stage" $(26 \%)$; sleep concerns (25\%); and "I have questions about my treatment options, medication, or my plan of care" $(24 \%)$. For validated measures, average scores were 2.9 for PHQ-4 (mild, >3.0), 6.2 for pain (mild, >4.0), 8.1 for fatigue (mild, $>6.0$ ), 7.6 for physical function (mild, >6.0). Notably, 59\% of patients with a PHQ-4 score of mild or higher had $\geq 5$ concerns compared with $34 \%$ of patients with a lower PHQ-4 score $(P=.0147)$. A comparison of individual patient PHQ-4 scores to their pain, fatigue, and physical function scores were not statistically significant $(P=.2634, .0676$, and .5697 , respectively). Conclusions: Patients experience multiple concerns and distress during their course of care. A tool that captures each patient's unique concerns may allow the care team to provide more holistic and personalized support for each patient, including earlier interventions of supportive care services. Additional studies are underway at multiple centers.

\section{AB2017-4. Optimizing Cancer Surveillance Protocols for Low-Grade Pediatric Brain Tumors Using Tumor Behavior Patterns}

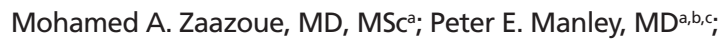

Mohammed AIMehdar, MSc; ; Nicole J. Ullrich, MD, PhD ${ }^{\mathrm{a}, \mathrm{c}}$;

Hormuzdiyar H. Dasenbrock, MD, MPH; ; and

Liliana C. Goumnerova, MD, FRCSC ${ }^{\mathrm{a}, \mathrm{c}}$

From ${ }^{a} B o s t o n$ Children's Hospital, ${ }^{b}$ Dana-Farber Cancer Institute, 'DanaFarber/Boston Children's Cancer and Blood Disorders Center, and 'Brandeis University

Background: Although low-grade brain tumors are among the most common pediatric neoplasms, optimal postoperative cancer surveillance remains nonstandardized. Current protocols involve arbitrarily chosen 
time-points based on expert opinion or retrospective data from small cohorts of patients. Methods: Patients with pediatric (aged 0-21 years) low-grade gliomas and glioneuronal tumors treated between November 1977 and March 2016 at Dana-Farber/Boston Children's Cancer and Blood Disorders Center were retrospectively reviewed, and data on timing of surveillance imaging and radiologic tumor outcomes were extracted. Multivariable cox proportional hazards model evaluated the association of covariates (tumor histology, location, age at diagnosis, and extent of resection) with time to recurrence. An algorithm was developed to analyze the efficacy of all possible combinations of surveillance protocols with reference to clinical milestones in the patients' timeline. A cost analysis was performed comparing the proposed protocols to the current practice, with imaging at $0,3,6,9,12,18,24,36,48,60,72,84,96$, 108, and 120 months. Results: A total of 576 patients were included, with a mean follow-up of 9.1 years, and 9,040 MR images were obtained (mean, 15.7 images/ patient). Tumor recurrence was detected radiographically in 313 patients (54.3\%), of whom 154 underwent reoperation. Of the recurrences, $62.6 \%$ occurred in the first 2 years of follow-up. The hazard ratio (HR) of recurrence was significantly higher in patients with neartotal resection ( $\mathrm{HR}, 1.85 ; 95 \% \mathrm{CI}, 1.08-3.18 ; \mathrm{P}=.03$ ) and subtotal resection or biopsy (HR, 1.36; $95 \% \mathrm{CI}$, $1.02-1.81 ; P=.04)$ compared with patients with gross total resection. Older age (11-21 years) was associated with decreased odds of sustaining a recurrence (odds ratio, $0.58 ; 95 \% \mathrm{CI}, 0.37-0.91 ; P=.019)$, but earlier time to recurrence $(\mathrm{HR}, 1.55 ; 95 \% \mathrm{CI}, 1.17-2.06$; $P=.003$ ) compared with patients aged 0 to 5 years. For all patients, an 8-image surveillance protocol at 0,3 , $6,12,24,36,60$, and 72 months (total cost, $\$ 13,672$ per patient) yielded comparative detection rates to the current 15-image protocol $(\$ 25,635)$. For gross-total resection patients, a 6-image protocol at $0,3,18,24,36$, and 60 months $(\$ 10,254)$ is sufficient. Conclusions: Posttreatment surveillance of pediatric low-grade brain neoplasms can be effectively performed using less frequent imaging, thereby improving cost-effectiveness, adherence to follow-up, and quality of life.

\section{Clinical Oncology \\ AB2017-5. Role of Chemoradiation Therapy in Patients With Initially Metastatic Anal Canal Cancer: A National Cancer Database Review}

Yasser A. Abdelwahab, MDa,b; Christel Rushing, MSc b; Manisha Palta, $\mathrm{MD}^{\mathrm{b}}$; Christopher Willet, $\mathrm{MD}^{\mathrm{b}}$; Bercedis Peterson, $\mathrm{PhD}^{\mathrm{b}}$; and Brian Czito, $\mathrm{MD}^{\mathrm{b}}$

From a National Cancer Institute, Cairo, Egypt, and ${ }^{b}$ Duke University Medical Center

Background: Although management of localized anal canal squamous cell carcinomas (SCCs) is well established, the role of chemoradiation (CRT) in the treatment of patients presenting with synchronous metastatic (stage IV) disease is poorly defined. We used a national cancer database to compare overall survival (OS) rates of patients with synchronous metastatic disease receiving CRT to the pelvis and chemotherapy versus those treated with chemotherapy alone. Methods: This retrospective study included adult patients with anal canal SCCs with synchronous metastasis who were diagnosed from 2004 to 2012. Multiple imputation and 2:1 propensity score matching were used to create a matched data set for testing this objective. The proportional hazards model was used to estimate the hazard ratio (HR) for the effect of treatment group on OS. Using only patients in the matched data set, the distribution of OS was estimated with the Kaplan-Meier method by treatment group. Results: Starting with an unmatched data set of 978 patients, 582 were selected into the matched data set: 388 in the CRT group and 194 in the chemotherapy-alone group. The HR for the group effect was 0.75 (95\% CI, 0.61-0.92), with a 2 -sided $P$ value of .006 . Median OS in the CRT group was 21 months (95\% CI, 17-24) versus 14 months (95\% CI, 12-18) in the chemotherapy group. The groups' respective 5-year OS rates were 0.23 (95\% CI, 0.18-0.28) and 0.14 (95\% CI, $0.07-0.21$ ). In a secondary analysis of the unmatched data set, we tested predictors of OS and found that men (vs women) and patients with visceral metastases (vs nodal metastases) had worse OS. Conclusions: In this largest series to date analyzing outcomes in patients with stage IV disease, CRT was associated with improved OS, whether delivered initially or following a period of initial systemic treatment versus chemotherapy alone. In view of the lack of prospective data in this setting, this evidence will help guide treatment approaches in this uncommon group of patients.

\section{AB2017-7. Downregulation of Stromal Cell Expression of Caveolin-1 Predicts Age, Tumor Size, and Lymph Metastasis in Patients With Nonmetastatic Triple- Negative Breast Cancer: Involvement of Caveolin-1 in Tumor Proliferation and Invasion}

Dongmei Gao, MDa; Tiennian Zhu, MD, PhDa; Frank Ma, MS'; and Xiaodong Feng, PhD, PharmD ${ }^{\mathrm{b}}$

From ${ }^{\mathrm{a} B e t h}$ une International Peace Hospital, and ${ }^{\mathrm{a} C a l i f o r n i a ~ N o r t h s t a t e ~}$ University College of Medicine

Background: Caveolin-1 (Cav-1) plays a critical role in tumor genesis and development; however its role in the development of triple-negative breast cancer (TNBC) is still not clearly delineated. TNBC is the most aggressive subtype of breast cancer, with poor prognosis and minimal response to hormonal therapy and target therapy. Currently there is no clearly defined 
biomarker to predict prognosis. We hypothesized that Cav-1 expression in TNBC stromal and epithelial cells predicts the prognosis of TNBC. This study provides the first evidence that downregulation of Cav-1 expression in TNBC stromal and epithelial cells correlates to the AJCC stages, tumor size, lymph metastasis, and younger age of patients with nonmetastatic TNBC. It also delineates the role of Cav-1 in TNBC cell proliferation, migration, invasion, and epidermal growth factor receptor (EGFR) signal transduction. Methods: Tissues of 51 cases of TNBC with documented estrogen receptor (ER), progesterone receptor (PR), HER2/neu negativity, and EGFR positivity were obtained from the surgical pathology department with the approval from the Institutional Review Board. These patients were not diagnosed with distant metastasis (stage IV) and did not receive chemotherapy or radiotherapy before surgery between 2009 and 2012 at Bethune International Peace Hospital. Pathologists were blinded to the experiments assessing and scoring the hematoxylin-eosin and immunohistochemistry tissue staining. Results: This study demonstrated for the first time that downregulation of Cav-1 expression in TNBC stromal cells was significantly correlated to the AJCC stages $(\mathrm{P}<.001)$, tumor size $(\mathrm{P}<.004)$, lymph metastasis $(\mathrm{P}<.004)$, and younger age $(\mathrm{P}<.026)$ of patients with nonmetastatic TNBC. It was previously reported that the expression of Cav-1 is generally upregulated in epithelial breast cancer cells and downregulated in stromal cells. Our study indicated that expression of Cav-1 was significantly downregulated in both epithelial cancer cells $(\mathrm{P}<.014)$ and stromal cells $(\mathrm{P}<.042)$ of nonmetastatic TNBC. In addition, in vitro data suggested that downregulation of Cav-1 expression significantly promote proliferation, migration, and tumor invasion of MDA-MB-436 cells of TNBC cell lines. EGF-induced phosphorylation of EGFR and activation of the RafMEK-ERK cascade were also upregulated by silencing Cav-1 expression in vitro. Conclusions: Our findings suggest that downregulation of Cav-1 can be a valuable biomarker for determining the prognosis of patients with nonmetastatic TNBC. Therefore, Cav-1 may be a potential novel strategy for TNBC treatment.

\section{AB2017-8. Interim Analysis of a Phase I Study of Enzalutamide in Patients With Advanced Hepatocellular Carcinoma}

James J. Harding, MDa; Robin K. Kelley, MD' Benjamin Tan, MD Khrystyna Uhlitskykh, BA; ; Carly Purcell, BA;

Kerri E. Muenkel, BA; ${ }^{a}$ Richard K. Do, MD, PhDa;

Marinela Capanu, PhDa; Carmen Ruiz, PhDd;

Mariam Rodriguez-Lee, $\mathrm{PhD}^{\mathrm{d}}$; John Wilton, $\mathrm{PhD}^{\mathrm{e}}$;

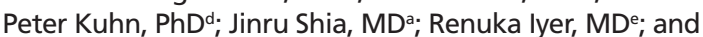

Ghassan K. Abou-Alfa, MDa
From ${ }^{a}$ Memorial Sloan Kettering Cancer Center, ${ }^{b}$ University of California, San Francisco, 'Washington University, 'USC David and Dana Dornsife College of Letters, Arts and Sciences, and 'Roswell Park Cancer Institute

Background: The androgen receptor (AR) is implicated in hepatocarcinogenesis. Preclinical models indicate that $\mathrm{AR}$ interference is deleterious to hepatocellular carcinoma (HCC) growth. Enzalutamide (ENZA) is a potent nonsteroidal inhibitor of AR with efficacy in a variety of solid tumors. Methods: This is a multicenter, NCCN-funded, phase I study of ENZA in patients with unresectable/metastatic HCC (ClinicalTrials.gov identifier: NCT02642913). A 3+3 dose de-escalation design with expansion cohort was used to establish safety and identify the ENZA recommended phase II dose. Toxicity was assessed using CTCAE v4.03 and efficacy with RECIST v1.1. AR expression by immunohistochemistry (IHC) and AR gene alterations by solution phase hybridization-based exon capture and DNA sequencing was determined on pretreatment tumors. Pretreatment and on-treatment circulating tumor cells (CTCs) were enumerated with a slide-based circulating epithelial assay and will be tested for AR expression. Steady state serum levels of ENZA and androgens will be determined on study closure. A sorafenib and ENZA cohort will begin upon completion of the ENZA expansion. Results: As of October 14, 2016, 9 patients were treated: median age, 70 years (range, 56-82); male, 56\%; hepatitis B virus, 33\%; hepatitis C virus, $0 \%$; Child Pugh A, $100 \%$; prior sorafenib, $100 \%$; 6 patients in the dose-finding cohort, 3 in the expansion cohort; all patients received ENZA, $160 \mathrm{mg} / \mathrm{d}$. No dose-limiting toxicity, treatment-related deaths, or case of treatment-related hepatic failure occurred. Grade $\geq 2$ treatment-related adverse events occurring in $\geq 2$ patients included fatigue, hypertension, hyponatremia, anemia, and thrombocytopenia. The 8-week overall response was 3 stable disease (SD), 4 progressive disease (PD), and 2 nonevaluable pending first interval imaging. At the time of writing, 3 patients remain on therapy, with 1 experiencing 24\% tumoral shrinkage, ongoing for 7 months. AR gene alterations were uncommon: 1 of 7 pretreatment tumors exhibited an AR V888E mutation. Of 7 pretreatment tumors, 5 tested positive for AR by IHC involving $40 \%$ to $90 \%$ of the specimen. Of 5 AR-positive patients, 3 had PD and 2 had SD. Among 2 AR-negative patients, 1 had $\mathrm{PD}$ and 1 had SD. CTCs were detected in 4 of 9 pretreatment samples (mean, $1.2 \pm 2.1 \mathrm{CTC} / \mathrm{mL}$ ) and 3 of 5 on-treatment samples (mean, $2.3 \pm 3.1 \mathrm{CTCl}$ $\mathrm{mL})$. Conclusions: ENZA at $160 \mathrm{mg} / \mathrm{d}$ orally is safe in patients with advanced HCC. Efficacy and biomarker evaluation is ongoing in the dose expansion. 


\section{AB2017-9. Impact of Granulocyte Colony-Stimulating Factor Use on Outcomes in Patients With Metastatic Breast Cancer Treated With Chemotherapy}

Luay Mousa, MDa; Michael Berger, PharmDa; Julie Stephens, MS'; Bhuvaneswari Ramaswamy, MDa; Nicole Williams, MDa;

Raquel Reinbolt, MD; ; Maryam Lustberg, $\mathrm{MD}^{\mathrm{a}}$; and Robert Wesolowski, MDa

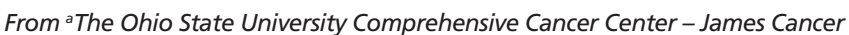
Hospital and Solove Research Institute, and ${ }^{b}$ The Ohio State University - Center of Biostatistics

Background: Neutropenia is a common toxicity of cytotoxic chemotherapy that results in dose delays and dose reductions. It is not clear whether use of granulocyte colony-stimulating factors (GCSFs) or decreasing chemotherapy dose to minimize the risk of neutropenia is associated with different clinical outcomes in patients with metastatic breast cancer (MBC). Methods: We reviewed the electronic medical records of patients treated for MBC with chemotherapy in the first- through third-line settings between May 2010 and April 2014 at the Ohio State University Comprehensive Cancer Center. Time to progression (TTP), progression-free survival (PFS), and overall survival (OS) were compared between patients who received GCSFs in the earliest line of treatment and patients who did not receive GCSF by using Kaplan-Meier (KM) curves and log-rank tests. We also compared ECOG performance status (PS) at the beginning and end of treatment in the 2 groups using either a chi-square or Fisher's exact test. Results: Of 169 patients who met the eligibility criteria, 55 (32.5\%) received $\geq 1$ dose of GCSF and 114 (67.5 $\%$ ) did not. More patients in the GCSF group were treated in the second or third line $(59.26 \%$ vs $40.55 \%$ in GCSF vs no GCSF groups, respectively). Median TTP was similar between patients in the GCSF and no GCSF groups (5.0 [95\% CI, 3.4-7.0] vs 5.2 months [95\% CI, 4.8-6.2], respectively; $P=.92)$, whereas PFS was not statistically different $(P=.87)$, with similar median PFS (5.0 [95\% CI, 3.4-5.9] and 5.2 months [95\% CI, 4.8-6.2], respectively). Median OS was also similar (14.6 vs 18.5 months in GCSF and no GCSF groups, respectively; $P=.581$ ). Among patients with ECOG PS of 0 to 1 at the beginning of chemotherapy, $20 \%$ in the GCSF group and $14 \%$ in the no GCSF group had an ECOG PS $>1$ at the end of chemotherapy $(P=.321)$. As expected, patients with ECOG PS $>1$ at the end of treatment had significantly shorter TTP and PFS compared with patients with ECOG PS 0 to 1 ( $P=.008$ and .003 , respectively), irrespective of GCSF use. Conclusions: The use of GCSF to maintain chemotherapy dose intensity for the treatment of MBC did not improve TTP, PFS, OS, or affect PS, although more patients in the GCSF group were treated in the second- and third-line settings. Our data suggest that reducing the dose of chemotherapy due to neutropenia rather than using GCSF in patients with MBC may be a reasonable strategy, but a prospective study is needed to confirm these conclusions.
AB2017-10. Percutaneous MRI-Guided Breast Biopsies: Tissue Marker Migration Rates and Associated Factors

Kimberly Funaro, MD; Andrew Prather, MD;

and R. Jared Weinfurtner, MD

From H. Lee Moffitt Cancer Center and Research Institute

Background: The objectives of this study were to identify rates and risk factors leading to tissue marker migration in patients undergoing MRI-guided percutaneous breast biopsy and to determine if tissue marker migration alters clinical management. Methods: All patients who had MRI-guided percutaneous breast biopsy at our institution between July 2010 and May 2015 were reviewed. Tissue marker migration was defined as final position of the tissue marker $>10 \mathrm{~mm}$ from the target site. Results: We identified 278 patients with 298 MRI-guided biopsies. Migration occurred in a total of 42 of 298 biopsies (14\%). Mostly fatty breast density was identified as an independent risk factor for tissue marker migration, occurring in 6 of 16 patients (38\%) versus 36 of $282(13 \%)$ for the other breast density categories $(P=.01)$. Biopsy target size was also identified as a risk factor for migration with 25 of 89 lesions $(22 \%)<10 \mathrm{~mm}$ resulting in migration versus 17 of $167(9 \%)$ for larger lesions $(P=.003)$. The presence of a hematoma demonstrated a trend toward increased migration, but this failed to reach statistical significance with migration occurring in 6 of 28 (21\%) of those patients with hematoma. When comparing migration rates of the 2 most common tissue marker shapes used at our institution, there was a trend toward more migrations with the SecurMark (Hologic, Inc., Marlborough, MA) infinity-shaped clip compared with the SecurMark buckle-shaped clip (20 of 94 [18\%] vs 13 of 123 [10\%], respectively; $P=.09)$. Clinical management was affected by clip migration in 10 cases (23\%), 5 of which required an ultrasound-guided clip placement, 2 required ultrasound-guided wire localization of the biopsy cavity, 2 required mammographic-guided wire localization of the cavity, and 1 required MRI-guided wire localization of the cavity. Conclusions: Migration of tissue markers at the time of MRI-guided percutaneous breast biopsy occurs with a similar rate to reported tissue marker migration rates in stereotactic and ultrasound-guided percutaneous breast biopsy, which can lead to changes in clinical management and may require performance of additional procedures. Low breast density and small target size are associated with increased rate of tissue marker migration. Clinical Relevance/Application: Migration of tissue markers after MRI-guided percutaneous breast biopsy is associated with low breast density and small target size, and may lead to additional procedures with possible alteration in clinical management. 
NCCN 22nd Annual Conference Abstracts

Outcomes and Health Services Research

AB2017-12. The NCCN-Flatiron Outcomes Database: Collaborative Evidence Development and Quality Improvement in Oncology

Vineeta Agarwala, MD, PhDa; Ben Birnbaum, PhD; James Gippetti, MSa; Laini Talcott, MHAa; Joshua Krauta; Bryan Bowsera; Joseph Delgadoa; Caroline Nightingalea; Michael Lvova; Nathan Nussbaum, MDa;

Dominique Connolly, RN, MA'; James Martineau, MS';

Katie Darius, $\mathrm{PhD}$; ; Kristen Fessele, $\mathrm{PhD}^{\mathrm{a}}$;

Amy Abernethy, MD, PhD; Joan McClure, $\mathrm{MS}^{\text {b; }}$, Lyn Fitzgerald, MJ'; and Robert Carlson, $\mathrm{MD}^{\mathrm{b}}$

From ${ }^{a}$ Flatiron Health, and ${ }^{b}$ National Comprehensive Cancer Network

Background: The NCCN Outcomes Database was created in 1997 to understand practice patterns, correlate them with patient outcomes, and inform clinical guidelines. The first version of the database was visionary in intent but difficult and costly to maintain, because it relied on distributed data abstraction at NCCN Member Institutions. NCCN recognized that a more sustainable model was needed, leveraging widespread availability of electronic health records (EHRs), a common data model, and the technology infrastructure to support data-sharing and analysis at scale. Methods: In 2015, NCCN initiated a collaboration with Flatiron Health (New York City, NY), a software company whose mission is to improve treatment and accelerate research in oncology. An interdisciplinary Flatiron team (oncologists, software engineers, informaticians, and database architects) is taking a systems engineering approach to the problem of clinical data curation. In collaboration with NCCN Guideline Panels, a common data model reflecting contemporary care was established across 6 vanguard tumor types. Scalable engineering pipelines were built to ingest and normalize discrete data fields from the EHR (eg, medication, laboratory values). To collect data from unstructured free-text, such as visit notes and pathology reports, Flatiron deployed a "technology-enabled" chart abstraction in which software assists clinically trained human abstractors in identifying data (eg, biomarker results). All data in the Outcomes Database are centrally deidentified and quality-controlled. Results: Flatiron has undertaken EHR integration with 2 large NCCN Member Institutions ( $>100,000$ total patient records) and relaunched the Quality \& Outcomes Database in Non-Small Cell Lung Cancer, Melanoma, Breast Cancer, and Colorectal Cancer at these centers. Data are refreshed by Flatiron quarterly, and processed patient-level data tables have been returned to each participating institution. Cohorts in additional tumor types will be launched throughout 2017. A secure Web portal supports database query and cohort visualizations. Quality measurement and guideline concordance solutions are being added to the portal, with benchmarking against peer institutions. Conclusions: NCCN collaboration with a technology group has enabled sustainable, standardized data capture and reduced chart abstraction and resource commitment from member centers. The NCCN-Flatiron Outcomes Database is one example of a data-sharing network that can support collaborative evidence development, quality improvement, and outcomes research.

\section{AB2017-13. Comparison of Clinical Outcomes Among Chelated Versus Nonchelated Patients With Myelodysplastic Syndromes: A Real-World Medicare Database Study}

Yanni Hao, PhD; ; Alex Fu, PhD ; Austin Coe, MPHa; Ying Qiu, PhD; ; Zhiyi Li, MBA, MAc; Becky Hanna, MPH'; Jackson Tang, MSc; Brian Elliott, MDa; and Carole Paley, MDa From ${ }^{a}$ Novartis Pharmaceuticals, ${ }^{b}$ Georgetown University Medical Center, and 'Asclepius Analytics

Background: Patients with myelodysplastic syndromes (MDS) often develop severe anemia and require periodic red blood cell (RBC) transfusions, which may lead to iron overload. Iron overload has been associated with increased mortality risk and shorter acute myeloid leukemia (AML)-free survival. Iron chelation therapy (ICT) may improve patient survival and quality of life. Deferasirox (DFX) is an oral chelator for the treatment of iron overload in patients with MDS. Methods: This retrospective cohort study used 100\% Medicare claims data from 2009 to 2013. Patients diagnosed with MDS using ICD-9 codes (238.72-238.76) were selected and entered the study after meeting a minimum transfusion threshold of either $10 \mathrm{RBC}$ transfusion episodes or 20 units. Patients were classified into ICT and nonICT cohorts, depending on whether ICT was received after study entry. Furthermore, patients who received DFX were classified into long ( $\geq 6$ months) or short $(<6$ months) duration groups based on the cumulative duration of DFX use post-study entry. Overall survival, AML-free survival, and cardiac event-free survival

\begin{tabular}{|c|c|c|c|c|c|c|}
\hline \multirow[b]{2}{*}{$\begin{array}{l}\text { 1-Year } \\
\text { Rate }\end{array}$} & \multicolumn{3}{|c|}{$\begin{array}{l}\text { All Eligible Patients } \\
\text { With MDS }(\mathrm{N}=6,796)\end{array}$} & \multicolumn{3}{|c|}{$\begin{array}{l}\text { Patients Treated } \\
\text { With DFX }(\mathrm{N}=583)\end{array}$} \\
\hline & $\begin{array}{c}\text { ICT Use } \\
\mathrm{N}=591 \\
(8 \%)\end{array}$ & $\begin{array}{c}\text { No ICT } \\
\text { Use } \\
\mathrm{N}=6,205 \\
(92 \%)\end{array}$ & $\begin{array}{c}P \\
\text { Value }\end{array}$ & $\begin{array}{c}\text { Long DFX } \\
\text { Duration } \\
(\geq 6 \mathrm{mo}) \\
\mathrm{N}=196 \\
(34 \%)\end{array}$ & $\begin{array}{c}\text { Short DFX } \\
\text { Duration } \\
(<6 \mathrm{mo}) \\
\mathrm{N}=387 \\
(66 \%)\end{array}$ & $\begin{array}{c}P \\
\text { Value }\end{array}$ \\
\hline Death & $27.6 \%$ & $66.5 \%$ & $<.01$ & $5.6 \%$ & $39.0 \%$ & $<.01$ \\
\hline $\begin{array}{l}\text { AML } \\
\text { progression } \\
\text { or death }\end{array}$ & $34.3 \%$ & $69.9 \%$ & $<.01$ & $15.3 \%$ & $44.4 \%$ & $<.01$ \\
\hline $\begin{array}{l}\text { Cardiac } \\
\text { event or } \\
\text { death }\end{array}$ & $78.9 \%$ & $93.7 \%$ & $<.01$ & $65.3 \%$ & $86.4 \%$ & $<.01$ \\
\hline
\end{tabular}

Abbreviations: AML, acute myeloid leukemia; DFX, deferasirox; ICT, iron chelation therapy; MDS, myelodysplastic syndromes. 
were compared between ICT and non-ICT patients and between long and short DFX duration patients using Kaplan-Meier (KM) analysis. Results: Approximately $591(8.7 \%)$ of the 6,796 patients with MDS who met the minimum transfusion threshold received ICT. The ICT cohort was younger (aged 77 vs 80 years; $P<.01)$, had a higher proportion of men ( $58 \%$ vs $52 \%$; $P<.01$ ), and had fewer comorbidities before cohort entry compared with the non-ICT cohort. Almost all ICT patients $(583 / 591 ; 98.6 \%)$ received DFX; 33.6\% $(196 / 583)$ and $62.4 \%(387 / 583)$ of them received $\geq 6$ months and $<6$ months of cumulative DFX treatment, respectively. The long DFX duration cohort had less comorbidity before cohort entry compared with the short DFX duration cohort. ICT patients experienced better survival outcomes than the non-ICT patients. In addition, this analysis also demonstrated that patients with $\geq 6$ months cumulative DFX had better survival outcomes than those with $<6$ months cumulative DFX. Survival rates by KM analysis are provided in Table 1. Conclusions: The proportion of Medicare patients treated with ICT among those with MDS who met a minimum threshold of RBC transfusions was low, despite ICT being associated with improved overall, AML-free, and cardiac event-free survival. Patients with $\geq 6$ months cumulative DFX use had significantly improved survival outcomes compared with those with $<6$ months cumulative DFX use.

\section{AB2017-14. Cost-Effectiveness of Pembrolizumab Versus Standard-of-Care Chemotherapy in First-Line Treatment of Patients With PD-L1-Positive Metastatic Non-Small Cell Lung Cancer}

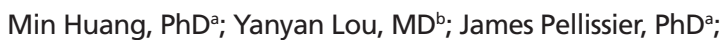
Thomas Burke, PhD; ; Frank X. Liu, PhDa; Ruifeng Xu, PhDa; and Vamsidhar Velcheti, MD ${ }^{c}$

From ${ }^{a}$ Merck \& Co., Inc., ${ }^{b}$ Mayo Clinic Cancer Center, and ${ }^{~ C C l e v e l a n d ~ C l i n i c ~}$

Background: The objective of the study was to evaluate the cost-effectiveness of pembrolizumab compared with standard-of-care platinum-based chemotherapy (SoC) in patients with previously untreated metastatic non-small cell lung cancer (NSCLC) whose tumors expressed programmed death ligand-1 (PD-L1; tumor proportion score [TPS], $\geq 50 \%)$. The analysis was conducted from a US third-party payer perspective. Methods: A partitioned-survival model was developed using data from patients in the KEYNOTE-024 (KN024) clinical trial. Patients in KN024 receiving SoC were able to switch to pembrolizumab on disease progression, which is consistent with real-world clinical practice, because pembrolizumab is currently indicated for the treatment of PD-L1-positive metastatic NSCLC after SoC in the United States. The model used Kaplan-Meier estimates of progression-free survival and overall survival for patients treated with pembrolizumab and SoC, with extrapolation based on fitted parametric functions and long-term registry data. Quality-adjusted life years (QALYs) were derived based on EQ-5D-3L data from KN024 using a time-to-death approach. Costs of drug acquisition/administration, adverse event management, subsequent therapy, and clinical management of metastatic NSCLC were included in the model. The base case analysis used a time horizon of 20 years. Costs and health outcomes were discounted at a rate of $3 \%$ per year. A series of one-way and probabilistic sensitivity analyses were performed to test the robustness of the results. Results: Base case results project a mean survival of 3.35 years for PD-L1positive (TPS $\geq 50 \%$ ) patients treated with pembrolizumab. For SoC, a mean survival time of 2.05 years was estimated. Expected QALYs were 2.60 and 1.55 for pembrolizumab and SoC, respectively. The incremental cost per life year (LY) gained with pembrolizumab versus SoC is $\$ 78,344 / \mathrm{LY}$ and the incremental cost per QALY gained is $\$ 97,621 / \mathrm{QALY}$, which is cost-effective per the WHO criteria of 3 times GDP per capita.

\section{AB2017-15. Healthcare Resource Utilization in Patients Receiving Pembrolizumab or Standard-of-Care Chemotherapy With Previously Untreated Metastatic Non-Small Cell Lung Cancer}

Min Huang, PhD; James Pelllissier, PhD; and Jane Liao, MA From Merck \& Co., Inc.

Background: This study aimed to evaluate healthcare resource utilization (HCRU) related to cancer management care within a clinical trial in patients with previously untreated metastatic non-small cell lung cancer (NSCLC). Methods: This study examined the HCRU data collected from patients enrolled in the KEYNOTE-024 (KN024) trial, a multicenter, worldwide, randomized phase III trial of pembrolizumab and standard-of-care platinum-based chemotherapy (SoC) in patients with previously untreated metastatic NSCLC with programmed death ligand-1 (PDL1)-positive (tumor proportion score [TPS] $\geq 50 \%$ ) tumors. The HCRU data captured non-study-related healthcare visits, (including visits for home-based hospice, home healthcare, nonstudy laboratory, radiation therapy $[\mathrm{RT}]$, nurse, primary care $[\mathrm{PCP}]$, and medical specialists), hospitalization (including admission and length of stay [LOS]), and emergency room (ER). We analyzed and compared the HCRU associated with patients treated with pembrolizumab versus SoC during the period between treatment initiation and disease progression. Results: Treated patients with $\geq 1$ contributing record (125 for pembrolizumab, 124 for $\mathrm{SoC})$ were included in the analyses. Higher HCRU 
NCCN 22nd Annual Conference Abstracts

was seen in patients treated with SoC compared with pembrolizumab in terms of hospitalization and most non-study-related healthcare visits. Average number of visits per patient-month was 0.40 (SoC) versus 0.23 (pembrolizumab) for medical specialist, 0.36 versus 0.20 for PCP, 0.41 versus 0.27 for laboratory, and 0.05 versus 0.02 for RT. Average hospital LOS was 2.70 (SoC) versus 1.79 days (pembrolizumab) per patientmonth. Average number of visits per patient-month to ER ( 0.00 vs 0.01$)$ and home-based hospice ( 0.02 vs 0.01) were similar between treatment arms. Average number per patient-month of home healthcare visits ( 0.03 vs 0.13$)$ and nurse visits $(0.13$ vs 0.28$)$ were lower in the SoC arm. Conclusions: Although within-trial HCRU may underestimate the HCRU outside a trial setting, within $\mathrm{KNO} 24$ the treatment of pembrolizumab showed numerically lower non-study HCRU compared with SoC in patients with previously untreated metastatic NSCLC with PD-L1-positive (TPS $\geq 50 \%$ ) tumors. These results can inform health economic models of treatment for NSCLC.

\section{AB2017-16. Evaluation of Cardiovascular Disease Among Patients With Multiple Myeloma Receiving Different Drug Classes of Antimyeloma Treatment in the United States \\ Daniel Lenihan, MDa; Ravi Potluri, PGDM ${ }^{\mathrm{b}}$. \\ Hemanth Kanakamedala, $\mathrm{BS}^{\mathrm{b}}$; Anandaroop Dasgupta, $\mathrm{PhD}^{\mathrm{b}}$; and Clara Chen, PhD, MSc \\ From ${ }^{a}$ Vanderbilt University, ${ }^{b}$ SmartAnalyst, Inc., and 'Bristol-Myers Squibb}

Background: Multiple myeloma (MM) is a disease of the elderly and is associated with a higher risk of cardiovascular disease (CVD); proteasome inhibitors (PIs) and immunomodulatory drugs (IMiDs) are its cornerstone therapies. The improved efficacy in patients receiving PI plus IMiD combination therapy $(\mathrm{PI}+\mathrm{IMiD})$ has resulted in an increase in PI+IMiD use in recent years. However, it has been hypothesized that patients receiving $\mathrm{PI}+\mathrm{IMiD}$ are at increased risk of developing CVD. We studied the prevalence of CVD in patients receiving $\mathrm{PIs}$, IMiDs, $\mathrm{PI}+\mathrm{IMiD}$ and chemotherapy regimens. Methods: Patients with an anti-MM treatment between July 2012 and September 2014 were identified in a large US claims database. Index date was the first claim for an anti-MM drug in this period, preceded by 6 months of continuous eligibility with no anti-MM treatment (baseline). CVDs of interest included cardiac arrhythmia, cardiac failure, stroke, ischemic heart disease, venous thromboembolism (VTE), angina, coronary atherosclerosis, and myocardial infarction. Patients were divided into subgroups based on class of anti-MM regimen and were assessed for prevalence of each CVD during the treatment period, which was compared with prevalence during baseline. Results: A total of 4,635 patients met the study eligibility cri- teria (57\% male; median age, 64 years; $55 \%$ with any one CVD at baseline; $42 \%$ with Charlson comorbidity index $\geq 2$ ). Patients receiving $\mathrm{PI}+\mathrm{IMiD}$ were younger than those receiving $\mathrm{PIs}$, IMiDs, or chemotherapy (mean age, 62 vs 67 years; $P<.001$ ), and more lived in the Northeast/South than elsewhere (21\% vs $15 \%$; $P<.001)$. The prevalence of CVD was generally higher during treatment than at baseline, with the largest increase in the PI+IMiD cohort and smallest in the chemotherapy cohort. Multivariable logistic regression analysis showed that patients receiving PI+IMiD were $68 \%$ more likely than those receiving PIs to develop a VTE $(P<.001)$ and $35 \%$ more likely than those receiving IMiDs to develop cardiac failure $(P=.048)$, after accounting for preexisting CVD as a prognostic factor. Conclusions: PIs and IMiDs may be associated with incremental occurrence of specific CVDs during anti-MM treatment, although $\mathrm{PI}+\mathrm{IMiD}$ may further increase such risk. Patients with preexisting CVDs were at a higher risk of experiencing the same CVD during treatment, which highlights the importance of evaluating baseline CVD, and a need for treatments that do not exacerbate CVD risk and are appropriate for patients with preexisting CVD.

\section{AB2017-17. The Psychosocial Impact of Lung Cancer- Related Stigma: Findings From the Cancer Experience Registry}

Joanne S. Buzaglo, PhD;; Melissa F. Miller, PhD, MPHa; Alexandra K. Zaleta, $\mathrm{PhD}^{\mathrm{a}}$; Jamese Johnson, $\mathrm{MPH}, \mathrm{CPH}^{\mathrm{a}}$; Linda House, RN, BSN, MSMa; Emily K. Porensky, PhDa; and Janine Cataldo, RN, $\mathrm{PhD}^{\mathrm{b}}$

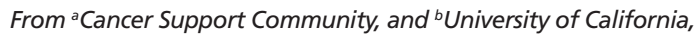
San Francisco

Background: Compared with other patients with cancer, those with lung cancer (LC) experience greater psychosocial distress and depression and report stigmatization because LC is strongly associated with smoking. The objective of this study was to characterize LC stigma, define the relationship with distress and depression, and identify covariates of stigma among LC survivors. Methods: To date, 33 LC survivors enrolled in the Cancer Experience Registry (CER) and answered stigma-related questions. Participants rated their agreement with 13 statements related to LC stigma $(\mathrm{O}=$ strongly disagree; $4=$ strongly agree). For distress, participants rated their concern $(0=$ not at all; $4=$ very seriously $)$ about 27 items, including a 4-item depression risk subscale. We examined (1) the association between stigma-related items and clinical and demographic variables (age, sex, time since diagnosis, LC type, recurrence, and presence of metastatic disease) and (2) the association between stigma and distress (sum of 27 items), using its square root, and risk for depression (score $\geq 6$ ) in multivari- 
ate linear and logistic regression adjusting for potential confounders. Results: The sample had a median age of 62 years and time since diagnosis of 2 years; diagnosis was $77 \%$ non-small cell LC; $41 \%$ experienced recurrence; $53 \%$ had metastatic disease. The highest ranked stigma-related items included (mean $\pm \mathrm{SD})$ : people assume smoking caused LC $(2.6 \pm 0.9)$, LC is viewed as self-inflicted $(2.8 \pm 1.4)$, and some people are uncomfortable around me because they associate $\mathrm{LC}$ with death $(2.3 \pm 1.2)$. LC type was associated $(P<.05)$ with several stigma-related items. Greater time since diagnosis was associated with being open to speak about one's LC diagnosis $(P=.037)$. There was a significant association ( $\beta[\mathrm{SE}] ; P$ value) between overall distress and feeling guilty because of having LC $(0.71[0.22] ; P=.007)$, being open to speaking about LC $(-0.99[0.37] ; P=.014)$, and trying to keep LC diagnosis a secret (0.80[0.31]; $P=.015)$. Feeling guilty was also associated with greater risk for depression (odds ratio, 4.7; 95\% CI, 1.5, 15.3). Conclusions: This study adds to the evidence that stigma impacts the health of patients with LC and has the potential to impact health-seeking behaviors. Importantly, this study focuses on LC survivors and suggests that type of LC and time since diagnosis are related to stigma. The CER continues to enroll patients with LC, and future analyses will refine and detail associations among stigma, distress, and psychological and physical outcomes in a larger sample.

\section{AB2017-19. Impact of Depression Treatment on Health- Related Quality of Life Among Adults With Cancer and Depression: A Population-Level Analysis}

Ami Vyas, PhD, MS, MBA; Zachary Babcock, MSA; and Stephen Kogut, PhD, MBA

From University of Rhode Island

Background: Cancer diagnosis in adults is often accompanied by negative impacts on mental health, changes in body function, persistent pain, and fear of cancer recurrence and death, which increase their risk of de-

\begin{tabular}{|lccc|}
\hline \multicolumn{1}{|c|}{ AB2017-19. Table 1. HRQOL in Adults With Cancer and Depression } \\
\hline $\begin{array}{l}\text { No Depression } \\
\text { Treatment }\end{array}$ & $\begin{array}{c}\text { Antidepressants } \\
\text { Only }\end{array}$ & $\begin{array}{c}\text { Psychotherapy } \pm \\
\text { Antidepressants }\end{array}$ \\
\hline $\begin{array}{l}\text { PCS, mean (SE) } \\
\text { MCS, mean (SE) }\end{array}$ & $40.72(0.85)$ & $39.87(0.87)$ & $40.97(1.55)$ \\
$\begin{array}{l}\text { Regression } \\
\text { estimates }\end{array}$ & $43.78(0.68)$ & $44.37(0.82)$ & $39.23(1.74)$ \\
$\begin{array}{l}\text { PCS (SE) } \\
\begin{array}{l}\text { Regression } \\
\text { estimates }\end{array} \\
\text { MCS (SE) }\end{array}$ & Ref & $-1.267(1.78)$ & $0.173(0.48)$ \\
\hline
\end{tabular}

Abbreviations: HRQOL, health-related quality of life; MCS, mental component summary; PCS, physical component summary.

asignificant at $P<.05$.

${ }^{b}$ Adjusted for age, sex, race, education, income, health insurance, cancer type time since cancer diagnosis, cancer remission, and chronic conditions. pression, thereby lowering health-related quality of life (HRQOL). We examined the association between depression treatment and HRQOL among US adults with cancer and depression. Methods: A total of 450 adults (weighted, 10.5 million) aged $\geq 18$ years with self-reported cancer and depression diagnoses were identified from the Medical Expenditure Panel Survey database from 2006 through 2013. Baseline depression treatment was categorized as antidepressants only, psychotherapy with or without antidepressants, and no reported use of antidepressants or psychotherapy. HRQOL was measured using SF-12 physical component summary and SF-12 mental component summary (MCS) scores. Adjusted ordinary least squares regression estimated the association between depression treatment and HRQOL. Results: $50 \%$ of the sample received antidepressants only, whereas $17 \%$ received psychotherapy with or without antidepressants. In bivariate analyses, the mean MCS score was lowest among those who received psychotherapy with or without antidepressants (39.23) compared with those receiving antidepressants only (44.37) and those with no reported use of either modality (43.78; $P<.05$; Table 1$)$. In multivariate analyses, no significant difference was seen in the HRQOL scores by type of depression treatment. Conclusions: Use of antidepressants or psychotherapy did not appear to favorably impact HRQOL among adults with cancer and depression. Quality of life is a priority health outcome in cancer treatment, yet our findings suggest that current clinical approaches to ameliorate depression in patients with cancer appear to be suboptimal.

\section{AB2017-20. Side Effects of Immune Checkpoint Therapy in Patients With Cancer Visiting the Emergency Department of a Comprehensive Cancer Center}

Myint Aung Win, MD; Kyaw Zin Thein, MD; Aiham Qdaisat, MD; Myat Min Han, MDa; and Sai-Ching Jim Yeung, MD, $\mathrm{PhD}^{\mathrm{a}}$

From ${ }^{a}$ The University of Texas MD Anderson Cancer Center, and ${ }^{b} T e x a s$ Tech University Health Sciences Center

Background: In the past few decades, immune checkpoint therapies (ICTs) targeting cytotoxic T-lymphocyte-associated protein 4 (CTLA-4), programmed cell death ligand-1 (PD-L1), and programmed cell death protein-1 (PD-1) were developed and are now approved for treatment of advanced stages of various cancer types. Because these ICTs enhance the immune response, they have specific side-effect profiles related to autoimmunity. Emergency department (ED) visits related to ICT side effects represent an integral part of healthcare delivery for patients with cancer receiving ICTs. Methods: We conducted a retrospective review of 628 consecutive patients treated with ICTs from March 1, 2011, to February 29, 2016, at MD Anderson Cancer Center. ICTs included ipilimumab, nivolumab, 
NCCN 22nd Annual Conference Abstracts

and pembrolizumab. Patient demographics and clinical parameters, including comorbidities, treatments rendered, date of first ICT dose, and subsequent ED visits, including the visits related to immune-related adverse events (irAEs), were collected and summarized. Analysis using chi-square, or Fisher's exact test where appropriate, was performed. Results: A total of 186, 154, and 109 patients received ipilimumab, nivolumab, and pembrolizumab, respectively, and 179 patients were treated with combination therapy either concurrently or sequentially. Diarrhea (14.5\%), colitis (7\%), dermatitis $(4.3 \%)$, and hypophysitis $(4.3 \%)$ were the most common irAEs leading to ED visits in patients who received ipilimumab. In the nivolumab group, diarrhea $(8.4 \%)$, pneumonitis $(7.1 \%)$, and dermatitis $(4.5 \%)$ were the leading causes of ED utilization. Diarrhea $(6.4 \%)$, pneumonitis $(4.6 \%)$, dermatitis $(4.6 \%)$, and colitis $(1.8 \%)$ were preeminent contributors in those treated with pembrolizumab. In patients who received combination therapy, diarrhea, colitis, and dermatitis were the most irAEs causing ED visits. Diarrhea, the most common irAE in our study, was significantly different among treatment groups $(P=.007)$. Thyroiditis, hypophysitis, and pancreatitis were also significantly different $(P=.008, .016$, and .049 , respectively). Conclusions: Diarrhea, colitis, pneumonitis, and dermatitis were the most common irAEs contributing to ED visits. Diarrhea, hypophysitis, thyroiditis, and pancreatitis were significantly different among the different patient groups. Because of the increasing use of ICTs for cancer treatment, healthcare professionals should be familiar with the distinct side effects of these agents to care for patients appropriately.

\section{Preclinical Oncology}

AB2017-21. Auranofin Prevents Progression of Human Pancreatic Ductal Adenocarcinoma

Mayrim Victoria Rios Perez, MDa; David Roife, MD; Bing Bing Dai, PhD'; Ya'an Kang, MD, PhD';

Xinqun Li, MD, PhD; ; Michael Pratt; and Jason B. Fleming, $\mathrm{MD}^{\mathrm{c}}$ From a Universidad de Puerto Rico Recinto de Ciencias Medicas, ${ }^{\circ}$ The University of Texas Health Science Center, and 'The University of Texas MD Anderson Cancer Center

This abstract is available at J Clin Oncol 2016;34(Suppl):Abstract 236.

\section{Quality Improvement}

AB2017-22. Applying Lean Principles and Predictive Analytics to Improve Patient Access, Decrease Wait Time, and Reduce Healthcare Delivery Costs at UCSF Helen Diller Family Comprehensive Cancer Center

Laurel Bray-Hanin, MA; ; Mohan Giridharadas, MBA, MS'; and Marisa Quinn, RN, MBA ${ }^{a}$

From a UCSF Helen Diller Family Comprehensive Cancer Center, and ${ }^{b}$ LeanTaaS
Background: UCSF Health's Adult Infusion Center at Mission Bay recently experienced 21\% growth in patient volumes and faced several operational challenges, such as regularly operating over maximum capacity and patient wait times of up to 2 hours during peak hours (10 AM-2 PM). Schedule gaps and staffing challenges as a result of appointments not being level-loaded across days of the week and hours of the day resulted in decreased staff and patient satisfaction and scheduling workflows dominated by exceptions to standard work, requiring Charge Nurse override. Methods: Given the varying appointment lengths and number of chairs, the number of permutations and combinations to accommodate patients with minimal idle chair times is very high and requires sophisticated mathematical calculations. For example, a 35-chair infusion center seeing 70 patients with varying treatment lengths can arrange them in $10^{\wedge} 106$ ways on a single day. Scheduling them "right" to increase patient access, decrease wait times, and reduce healthcare delivery costs is next to impossible without sophisticated mathematical modeling. Center staff hypothesized that changing the infusion schedule from an acuitybased to a length-of-treatment-based template and rebalancing medical oncologists' schedules would address their operational challenges. UCSF Health deployed LeanTaaS's iQueue for Infusion Centers (Santa Clara, CA) solution at its center at Mission Bay to create optimized infusion scheduling templates. Results: The staff at the Adult Infusion Center at Mission Bay set goals of reducing patient wait times by $25 \%$, having continuous patient flow to support staff break times, and enhancing patient safety and satisfaction. By deploying iQueue for Infusion Centers, the center was able to decrease both wait times during peak hours by $31 \%$ and average wait time overall by $26 \%$; reduce hours over capacity by $42 \%$; and reduce peak demand by $8 \%$. Based on these results, UCSF Health is implementing the iQueue for Infusion Centers solution at its 4 other centers. Conclusions: A growth in patient volume at UCSF Health's Adult Infusion Center at Mission Bay and the lack of coordination between infusion and practice templates artificially created peak demand times for its infusion chairs. By applying lean principles, predictive analytics, and sophisticated mathematical modeling, UCSF Health has flattened chair use, increased patient access, decreased wait times, and reduced healthcare delivery costs.

\section{AB2017-23. Development of Cancer Survivorship Clinical Dashboard}

Dori Klemanski, DNP, CNP, and Maryam Lustberg, MD, MPH

From The Ohio State University Comprehensive Cancer Center -

James Cancer Hospital and Solove Research Institute 
Background: American oncology institutions regularly evaluate their cancer survivorship programs to ensure compliance with the Commission on Cancer (CoC) program standards. However, there is no national consensus and action plan regarding cancer survivorship benchmarking. In 2012, our institution created a cancer survivorship quality dashboard to monitor and improve the structure and quality of cancer survivorship care. Methods: A multidisciplinary team of stakeholders met to develop a quality dashboard for cancer survivorship clinical programming using the define-measure-analyzeimprove-control (DMAIC) principles from Lean Six Sigma. An environmental scan exposed the lack of existing measures and data reports within the institution related to cancer survivorship care. Possible outcome measures were characterized by (1) feasibility (2) shortand long-term goals, (3) impact on clinical practice, and (4) institutional performance objectives. A prioritization matrix was used to determine the impact factor of each idea. Results: The initial dashboard was created in 2013 to track clinical volume, referring provider satisfaction, and demographics. Subsequent quarterly dashboards became more complex with the inclusion of comparison data by clinical specialty (multidisciplinary and disease-specific survivorship clinics, psychosocial oncology, and oncology rehabilitation), patient satisfaction, utilization of patient education, and clinical data such as wait times, referrals, and survivorship care plan provision. The dashboards are distributed quarterly to providers and executive leaders. Barriers to implementation included (1) coordination and collaboration among stakeholders from clinical teams, information technology, and the quality department, (2) limited data automation from the electronic health record, and (3) ensuring the accuracy of large data sets. Conclusions: Our institution customized a quality improvement monitoring process to scrutinize clinical performance. The dashboard is useful for measuring program metrics, demonstrating compliance with CoC standards, and obtaining resources for programmatic expansion.

\section{AB2017-24. Supportive Oncology Care: An NCCN Online Educational Series}

Julia R. Trosman, PhD, MBA; ; Betty Roggenkamp, BA; Stephanie Merce Boecher, RN, OCN, MSN;

Amy Scheu, MSH, CHPCA; Patricia A. Robinson, MDd;

Frank J. Penedo, PhD'; Sofia F. Garcia, PhD'; Teresa Lillis, PhD; Rosa Berardiq; Javier Macias, BA ${ }^{\text {h; }}$ James Gerhart, $\mathrm{PhD}^{\text {f; }}$

Pam Khosla, MDi; Shelly Lo, MDd; Lauren Wiebe, MD;; William Dale, MD PhD; ; Sheetal Mehta Kircher, MD'; Carol Newsom, RNm; Carol A. Rosenberg MD;

Catherine Deamant, $\mathrm{MD}^{\mathrm{n}}$; and Christine B. Weldon, $\mathrm{MBA}^{\mathrm{a}}$ From ${ }^{a}$ The Center for Business Models in Healthcare, ${ }^{b}$ On the Side Design, 'Advocate Health Care, ${ }^{d}$ Loyola University Medical Center, eNorthwestern University, ${ }^{f}$ Rush University Medical Center, ${ }^{9}$ The Coleman Foundation,

${ }^{h}$ The Leukemia \& Lymphoma Society, 'Mount Sinai Hospital, 'NorthShore
University HealthSystem, ${ }^{k}$ City of Hope, 'Robert H. Lurie Comprehensive Cancer Center of Northwestern University, ${ }^{m}$ Mercy Hospital and Medical Center, and "Journey Care

Background: Supportive oncology is integral to patient care, as indicated by evidence and recommendations in the NCCN Clinical Practice Guidelines in Oncology (NCCN Guidelines) for Supportive Care, the Commission on Cancer standards (2.4 Palliative Care Services, 3.2 Psychosocial Distress Screening, 3.3 Survivorship Care Plan), ASCO guidance, and the Institute on Medicine's 2013 Report on Delivering HighQuality Cancer Care. The Coleman Supportive Oncology Collaborative (CSOC) is focused on improving supportive care through tools, processes, and training of healthcare professionals. Methods: The Coleman Foundation funded a collaborative of $\geq 30$ faculty from 25 institutions (academic, community, and safety net hospitals; cancer support centers; palliative/hospice organizations; and patient advocacies) to review existing supportive oncology training for content, availability, and cost. They developed a unique supportive oncology online educational series. Results: Although some supportive oncology training is available, it lacks $\geq 1$ attributes: free CME/CEU credit, $<15$ minutes to complete, and online availability. Through suggestions from $>100$ participants in the CSOC and review of existing guidelines and literature, 26 training modules were identified: palliative (11), distress (6), and survivorship (9). Each module was developed by $\geq 2$ expert faculty in a standard format, including references and links to additional training, guidelines, and literature. NCCN scientists reviewed and edited each module. Faculty for each module recorded audio, which was technically aligned to video and graphics in an MP4 (movie) format. The NCCN Education Portal then published the training at https://education.nccn.org/supportive-oncology-care, with free CME/CEU credit offered. Conclusions: Various forms of supportive oncology training exist for healthcare professionals, but they lack needed attributes. The CSOC developed 26 concise supportive oncology training modules for NCCN's online educational series, with CE credit available at no cost to participants. Feedback from educational series trainees will inform effectiveness of the CSOC modules and identify future training needs.

\section{Young Investigator Awards}

\section{Correlative/Genomic}

\section{AB2017-25. Quantification of FLT3 Internal Tandem Duplication Status by Amplicon High-Throughput Sequencing Produces Superior Estimates of Patient Survival Versus Capillary Electrophoresis}

James Stewart Blachly, MD; Ann-Katherin Eisfeld, MDa; Jessica Kohlschmidt, PhD a,b; Deedra Nicolet, PhD ${ }^{\mathrm{a}, \mathrm{b}}$; Shelley Orwick ${ }^{\mathrm{a}}$; 
NCCN 22nd Annual Conference Abstracts

Jonathan E. Kolitz, MD; Bayard L. Powell, MD'; Eunice Wang, MDe; Richard M. Stone, MD; ; David Lucas, PhD; ;ing Wen, MBa; Krzysztof Mrozek, MD, PhDa; Clara D. Bloomfield, MD; and John C. Byrd, MDa

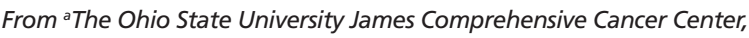
${ }^{b}$ The Alliance Statistics and Data Center, 'Hofstra North Shore-Long Island Jewish School of Medicine, 'Wake Forest Baptist Comprehensive Cancer Center, 'eRoswell Park Cancer Institute, and 'Brigham and Women's Hospital/Dana-Farber Cancer Institute

Background: Acute myeloid leukemia (AML) is the most commonly diagnosed leukemia and has a poor prognosis, with 5-year overall survival (OS) rates among adults of only $25 \%$. Patients harboring FLT3 internal tandem duplication (FLT3-ITD) generally fare worse. For patients with normal karyotype $(\mathrm{CN}$ AML), the occurrence of FLT3-ITD confers a lower likelihood of complete response and a higher risk of relapse and death; patients who lack wild-type (WT) FLT3 or show a high ratio of ITD to WT FLT3 have especially poor outcomes. Traditionally, ITD status has been determined by amplification of a region of FLT3 and fragment size analysis by capillary electrophoresis. Upward shift on the electropherogram (EPG) implies an addition of genetic material. By comparing areas under the curve (AUCs) of EPG traces, a FLT3 ratio is computed that can provide an estimate of patient outcome. Because this method is laborious, we tested whether high-throughput sequencing (HTS) could provide a more accurate measurement of the FLT3 ratio. Methods: We identified 1,196 patients from CALGB clinical trials who previously had FLT3-ITD analysis via EPG. The FLT3-ITD ratio was computed by dividing the AUC of the larger (ITD) fragment into the AUC of the smaller (WT) fragment. For HTS analysis, we prepared TruSeq Custom Amplicon libraries (Illumina, San Diego, CA) and sequenced these on a MiSeq instrument. Aligned reads were passed to Pindel and then to a bespoke bioinformatics tool. Results: Of 1,196 patients, 301 (25\%) had significant FLT3-ITD allelic ratios by established criteria, and 895 (75\%) were FLT3 WT or had allelic ratios below the significance cutoff. Our HTS data, using a read ratio (RR) cutoff of 0.01 , called 49 (4\%) of EPG-identified ITD cases WT and 28 (2\%) of EPG-identified WT cases as ITD, but still provided more prognostic information. Using Akaike Information Criterion (AIC; lower is better), the HTS method was superior to the EPG method for disease-free survival (DFS) and OS for younger $(2,526.74$ vs $2,530.96 ; n=353$, and $2,964.30$ vs $2,965.32 ; n=426$, respectively) and older (612.67 vs $615.94 ; n=91$, and $1,084.76$ vs $1,085.25$; $\mathrm{n}=145$, respectively) patients with $\mathrm{CN}-\mathrm{AML}$, and DFS and OS for older patients $(1,370.70$ vs $1,373.27$; $n=170$, and $3,068.66$ vs $3,070.20 ; n=332$, respectively) and OS for younger patients $(5,490.18$ vs $5,491.60)$ in the total cohort. The sole exception was DFS in all patients aged $<60$ years $(3,999.37$ vs $3,997.27 ; n=494)$. Conclusions: Our results show for the first time in a large patient cohort that assessment of FLT3-ITD allelic ratio using HTS is a superior predictor of survival compared with standard EPG analysis.

\section{Outcomes and Health Services Research \\ AB2017-26. A Multimodal Intervention to Address Sexual Dysfunction in Hematopoietic Stem Cell Transplant Survivors: A Feasibility Study \\ Areej El-Jawahri, MD; Sarah Fishman, BA; Julie Vanderklish, NP; Lara Treager, PhD; Don Dizon, MD; and Jennifer Temel, MD From Massachusetts General Hospital Cancer Center}

Background: For allogeneic hematopoietic stem cell transplant (HCT) survivors, sexual dysfunction is a common, yet rarely discussed, long-term complication affecting patients' quality of life (QOL) and intimacy. In this feasibility study, we aimed to systematically screen HCT survivors for sexual dysfunction and assess the feasibility of a multimodal sexual dysfunction intervention. Methods: Transplant clinicians systematically screened all HCT survivors attending their routine follow-up clinic appointments at Massachusetts General Hospital Cancer Center for sexual dysfunction causing distress. Patients were deemed eligible if they were $\geq 3$ months post-HCT and screened positive for sexual dysfunction causing distress. The intervention entailed assessing and treating sexual dysfunction by a transplant clinician and referring patients with more complicated needs to the sexual health clinic. The primary end point was feasibility. The proposed intervention will be deemed feasible if (1) $\geq 75 \%$ of patients screening positive for sexual dysfunction causing distress agreed to participate; and ( 2 ) $\geq 80 \%$ of participants attended a minimum of 2 intervention visits. Results: Of the 126 patients screened, 34\% (43/113) screened positive for sexual dysfunction causing distress. Overall, $93 \%$ of patients (40/43) who screened positive consented and enrolled in the study. Median age of participants was 50 years (range, 24-74 years) and 50\% (20/40) were female. Of the 40 patients enrolled, 12 completed the 6-month study period and $100 \%$ attended at least 2 intervention visits. The primary causes of sexual dysfunction addressed in men were erectile dysfunction $(65 \%)$, intimacy $(20 \%)$, decreased libido $(10 \%)$, and decreased arousal $(5 \%)$. The primary causes of sexual dysfunction addressed in women were pain with intercourse $(70 \%)$, vaginal graft-versus-host disease (20\%), and body image concerns (15\%). Psychological concerns were important secondary causes of sexual dysfunction addressed in 30\% of men and $20 \%$ of women 
Conclusions: Sexual dysfunction is a prevalent problem affecting more than one-third of HCT survivors. The proposed intervention appears feasible, with $>90 \%$ of eligible patients successfully participating and showing encouraging compliance with intervention visits. Future efforts will assess the preliminary efficacy of the intervention in improving sexual function and patients' overall QOL and mood.

\section{Clinical Oncology}

\section{AB2017-27. Long-Term and Cardiometabolic Effects of} Anti-PD-1/PD-L1 Agents

Douglas B. Johnson, MD; Javid J. Moslehi, MD;

Katy E. Beckermann, MD, PhDa; Emily H. Castellanos, $\mathrm{MD}^{\mathrm{a}}$;

Megan H. Pollack, PharmDa; Leora Horn, MDa;

David Chism, MD; ; Igor Puzanov, MD

Christine M. Lovly, MD, PhD; ; Debra L. Friedman, MDa; and Jeffrey A. Sosman, MD

From ${ }^{a}$ Vanderbilt University Medical Center, ${ }^{b}$ Roswell Park Cancer Institute, and 'Robert H. Lurie Comprehensive Cancer Center of Northwestern University

Background: Agents blocking programmed death-1/ ligand-1 (aPD-1) induce durable responses in many patients with cancer. Long-term toxicities of these agents, including cardiac and central nervous system (CNS) effects, are not well-characterized. Methods: We evaluated patients with various cancers with $\geq 2$ year survival on aPD-1 treatment. Retrospective chart review was conducted to capture short- and long-term toxicities, changes in weight and metabolic parameters, and cancer outcomes. Acute and chronic cardiac toxicities were assessed. Results: In the long-term cohort, we identified 57 patients with a median of 36.7 months follow-up after aPD-1 treatment initiation, including 31 with melanoma, 12 with non-small cell lung cancer (NSCLC), and 14 with renal cell carcinoma (RCC). Of these, only 4 died during the study period, and 19 (33\%) required additional therapies after aPD-1 progression (13\% with melanoma, 27\% with NSCLC, $79 \%$ with RCC). Chronic drug-related toxicities included low-grade arthralgias (14\%) and endocrinopathies (19\%, primarily hypothyroid and all with ongoing hormone replacement). Only 2 patients continued aPD- 1 beyond 3 years; one developed pneumonitis at 3.5 years on aPD-1. Of 8 patients with CNS radiation, 4 had radiation necrosis on imaging, although only 1 developed symptoms (seizures, progressive functional decline). Among living patients, no differences were observed in ECOG functional status between baseline and last follow-up. Between baseline and 2 years, no differences in blood pressure, weight, or glucose were observed. No long-term cardiac events were identified, but 2 acute cases of fatal myocarditis occurred in patients treated with ipilimumab/ nivolumab. Quality-of-life and further cardiac data will also be presented. Conclusions: Long-term toxicities related to aPD-1 rarely resulted in functional decline and most commonly presented as low-grade arthralgias, asymptomatic endocrinopathies, and/or radiation necrosis of variable severity. No long-term cardiometabolic signals were identified in this cohort, although further investigation into the cardiac effects of aPD-1 and interactions with radiation therapy is warranted.

\section{Correlative/Genomic \\ AB2017-28. Prognostic Utility of a Multigene Signature (the Cell Cycle Proliferation Score) in Patients With Renal Cell Carcinoma After Radical Nephrectomy}

Todd M. Morgan, MDa; Rohit Mehra, MDa; Placede Tiemeny, PhD ${ }^{\text {; }}$ J. Stuart Wolf, MD; ; Steven Stone, PhD; ${ }^{\text {; }}$ Chin-Lee Wu, MD; and Adam S. Feldman, MD ${ }^{c}$

From a University of Michigan Comprehensive Cancer Center, ${ }^{b}$ Myriad Genetics, Inc., cMassachusetts General Hospital Cancer Center

Background: There are currently no renal cell carcinoma (RCC) biomarkers routinely being used in the clinic, and prognostic nomograms rely almost entirely on tumor size, stage, and patient age. There is a critical need for improved prognostic discrimination given the increasing awareness that some patients may be managed with active surveillance, whereas others with higher-risk disease might benefit from adjuvant therapy

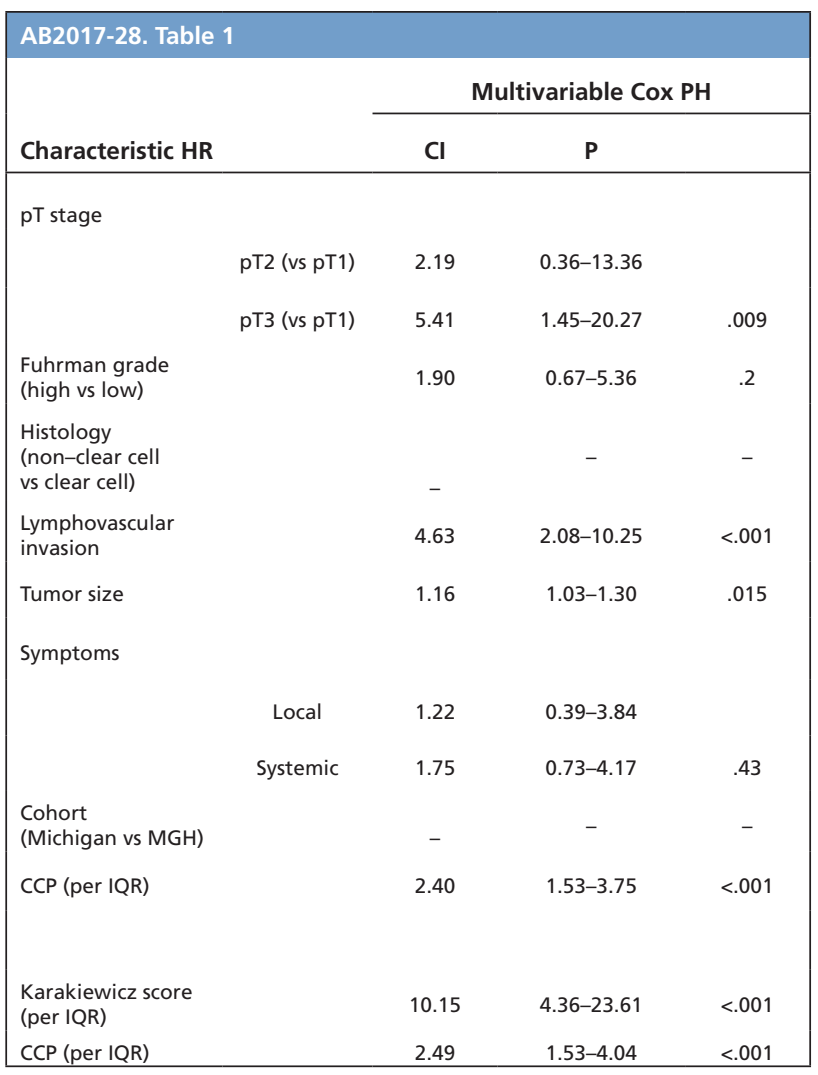

Abbreviations: $\mathrm{CCP}$, cell cycle proliferation; HR, hazard ratio; IQR, interquartile range; MGH, Massachusetts General Hospital; PH, proportional hazards. 
after surgery. We hypothesized that a previously developed multigene proliferation signature would predict long-term oncologic outcomes in surgically resected RCC. Methods: The cell cycle proliferation (CCP) score was derived after radical nephrectomy in $565 \mathrm{pa}$ tients who were treated at 1 of 2 institutions from 2000 to 2009 for clear cell, papillary, or chromophobe RCC with localized disease (NOMO). The primary end point was disease-specific survival (DSS), and disease recurrence (local or metastatic) was a secondary end point. Association with outcomes was evaluated by Cox proportional hazards survival analysis. CCP score was then compared with the Karakiewicz nomogram for prediction of recurrence and survival. Results: Patients had a median age of 61 years, and $58 \%$ of the cohort had pT1 disease. Patient data were censored at 5 -years of follow-up, by which time 68 patients (12\%) experienced recurrence and 32 (6\%) died of disease. In the development cohort, the median CCP score was 0.095 (interquartile range [IQR], 0.50-0.60). In univariable analysis, CCP score was a significant prognostic variable for recurrence $(P<.001)$ and DSS $(P<.001)$. After adjusting for clinical variables, including tumor size, stage, and grade, the CCP score hazard ratio (HR) for recurrence was 1.60 per IQR (95\% CI, 1.17-2.19), and for DSS was 2.40 (95\% CI, 1.53-3.75; Table 1). There was no interaction between CCP and any clinical variable. Controlling for the Karakiewicz nomogram score in the second model gave a similar association between CCP and DSM (HR, 2.49; 95\% CI, 1.53-4.04). Conclusions: The CCP score appears to be a significant and independent predictor of key long-term oncologic outcomes in patients who have undergone nephrectomy for RCC, providing prognostic information beyond what is available from clinical parameters. With further validation, the CCP score may have utility in the clinical management of patients with RCC.

\section{Outcomes and Health Services Research}

\section{AB2017-29. Partnered Status and Receipt of NCCN} Guideline-Concordant Colon Cancer Care

Christine M. Veenstra, MD, MSHP; Sarah T. Hawley, PhD, MPH; Mousumi Banerjee, PhD; and Jennifer J. Griggs, MD, MPH

From University of Michigan Comprehensive Cancer Center

Background: It is unknown whether receipt of guideline-concordant care differs among married/partnered and unpartnered patients. Because NCCN Guidelines for the treatment of stage III colon cancer are clear, comprehensive, and evidence-based, they provide an excellent case for investigating this issue. The NCCN Guidelines recommend 6 months of adjuvant chemotherapy and prefer multiagent chemotherapy over single-agent regimens. Methods: Sociodemographic, clinical, and treatment data were abstracted from medical records of patients with resected stage III colon cancer between 2008 and 2015. Patients were sampled from 3 sites: the University of Michigan and 2 community practices within the Michigan Cancer Research Consortium National Clinical Oncology Research Program. Outcome measures were (1) receipt of any adjuvant chemotherapy, (2) receipt of multiagent versus single-agent chemotherapy, and (3) completion of 6 months of adjuvant chemotherapy. Partnered status was the primary independent variable. We assessed associations between patient variables, partnered status, and outcome measures using multivariable logistic regression. Results: We report interim analyses of 317 of 450 patients; $60 \%$ were married/living with a partner, and $77 \%$ received any adjuvant chemotherapy. $\mathrm{Pa}$ tients aged $>70$ years were significantly less likely than those aged $<60$ years to receive adjuvant chemotherapy (odds ratio [OR], 0.05; 95\% CI, 0.01-0.24), and patients with $\geq 2$ comorbid conditions were significantly less likely than those with 0 or 1 comorbid conditions to receive adjuvant chemotherapy (OR, 0.32; 95\% CI, 0.12-0.88). Among those who received adjuvant therapy, $83 \%$ received multiagent chemotherapy. Women were significantly more likely than men to receive multiagent chemotherapy (OR, 3.40; 95\% CI, 1.41-8.21), and patients aged $>70$ years were significantly less likely than those aged $<60$ years to receive multiagent chemotherapy (OR, 0.27; 95\% CI, 0.11-0.69); 31\% of patients completed 6 months of adjuvant therapy. Partnered patients were significantly more likely than unpartnered patients to complete chemotherapy (OR, 2.10; 95\% CI, 1.03-4.29). Conclusions: In this interim analysis, most patients received adjuvant, multiagent chemotherapy in concordance with NCCN Guidelines. Early cessation of chemotherapy was common, and partnered patients had twice the odds of completing chemotherapy compared with unpartnered patients. Unpartnered patients may require additional supports to ensure guideline-concordant care and achieve the same clinical outcomes as partnered patients. 